\title{
Respiratory gas exchange in the assessment of patients with impaired ventricular function
}

\author{
DAVID P LIPKIN, JOHN PERRINS, PHILIP A POOLE-WILSON
}

\author{
From the Cardiothoracic Institute and National Heart Hospital, London
}

SUMMARY Respiratory gas exchange on exercise was evaluated as a non-invasive method of assessing patients with heart failure. Twenty four men (age 28-72) with symptomatic chronic stable heart failure (New York Heart Association class II-III) and ten controls aged 36-70 were studied. During treadmill exercise oxygen consumption and carbon dioxide production were measured continuously by analysis of mixed expired gas with a computerised mass spectrometer. The anaerobic threshold was defined as the oxygen consumption at which carbon dioxide production increased disproportionately in relation to oxygen consumption.

Oxygen consumption was stable at rest and increased on exercise, reaching a steady state within three minutes of any change in workload. The measurements of maximum oxygen consumption at the end of exercise and of anaerobic threshold were reproducible (retest reliability coefficients $90 \%$ and $91 \%$ respectively). There were significant differences in maximum oxygen consumption between functional classes. Similarly, there were significant differences in anaerobic threshold between classes, though there was considerable overlap.

Measurement of oxygen consumption and anaerobic threshold provides an objective noninvasive assessment of patients with heart failure.

The physiological stress of exercise has been widely used to evaluate the symptoms and cardiac function of patients with heart disease. ${ }^{1-4}$ It should be possible to assess the severity of heart disease and determine functional capacity by measuring a patient's response to exercise. Left ventricular function at rest as assessed by angiocardiography, echocardiography, or radionucleotide techniques correlates poorly with functional class. ${ }^{4}$ Exercise haemodynamic monitoring is invasive and is not without risk. ${ }^{5}$ Furthermore haemodynamic variables measured at rest or on exercise do not relate closely to exercise capacity, in patients with severe heart failure. ${ }^{26}$

Recently, impaired cardiac function has been assessed by measuring oxygen consumption during exercise. ${ }^{346-8}$ Patients with congestive cardiac failure have a reduced maximum aerobic exercise capacity because of inadequate transport of oxygen to

Requests for reprints to Dr D P Lipkin, The Cardiothoracic Institute, 2 Beaumont Street, London W1N 2DX.

Accepted for publication 10 June 1985 working skeletal muscle ${ }^{7}$ Lactic acid is generated by anaerobic metabolism in skeletal muscle. Buffering of lactic acid by bicarbonate generates carbon dioxide in excess of that resulting from oxidative metabolism. An aerobic-anaerobic threshold was first postulated over 20 years ago, ${ }^{9}$ and is thought to be reflected by a sharp increase in plasma lactate. Measurement of anaerobic threshold has been proposed as an objective means of assessing aerobic capacity. ${ }^{10-13}$ The clinical importance of such a measurement in cardiac patients is less certain. We used a mass spectrometer linked to a computer to analyse mixed expired air and thus determine maximum oxygen consumption and anaerobic threshold in patients with cardiac failure. We investigated the reproducibility of the test and the relation of oxygen consumption and anaerobic threshold to the severity of heart failure.

\section{Patients and methods}

We studied patients who had had stable symptomatic heart failure for more than three months with exercise intolerance due to dyspnoea, fatigue, or 
aching legs. In addition patients had cardiomegaly, defined as a cardiothoracic ratio $>55 \%$ on chest radiograph, and left ventricular ejection fraction $\leqslant 45 \%$ on radionucleotide angiography or echocardiographic study. Twenty four men (mean age was 50 years, range 28-72) entered the study. The cause of heart failure was coronary artery disease in 15 , dilated cardiomyopathy in six, hypertensive heart disease in two, and valvar regurgitation in one. Individual drug therapy was recorded. Patients with myocardial infarction during the previous six months, evidence of myocardial ischaemia or ventricular arrhythmias on exercise testing, obstructive valvar heart disease, pulmonary disease (documented by pulmonary function tests), or an inability to exercise for any reason other than dyspnoea or fatigue were excluded from the study.

Ten men (mean age 48 years, range 36-70) who were free of heart disease on the basis of history, physical examination, and 12 lead electrocardiogram were also studied. All patients and controls gave written consent to the study.

Functional class was assessed by modified criteria of the New York Heart Association ${ }^{14}$ : (I) No undue symptoms on strenuous exercise; (II) able to walk indefinitely without slowing pace and (a) climb two flights of stairs or (b) unable to climb two flights of stairs; (III) walk at a reduced pace on the flat (a) indefinitely at a reduced rate or (b) have to stop; (IV) symptoms at rest. Functional class was also assessed by a specific activity scale (1981). ${ }^{15}$ Patients were asked to fill in a standardised questionnaire on their symptoms.

\section{EXERCISE TEST}

All patients underwent at least three exercise tests over two to 14 days. In 10 patients four exercise tests were performed. Patients exercised three hours after a light meal, at the same time of day, and three hours after a last dose of any vasodilator therapy.

Each patient underwent progressive treadmill exercise testing which followed a heart failure protocol (stage $1,1 \mathrm{mph}(1.6 \mathrm{~km} / \mathrm{h})$ with $0 \%$ gradient; stage $2,1.5 \mathrm{mph}(2.4 \mathrm{~km} / \mathrm{h})$ with $0 \%$ gradient; stage 3, $2 \mathrm{mph}(3.2 \mathrm{~km} / \mathrm{h})$ with $0 \%$ gradient; stage $4,2.5$ $\mathrm{mph}(4 \mathrm{~km} / \mathrm{h})$ with $0 \%$ gradient; stage $5,2.5 \mathrm{mph}$ with $2.5 \%$ gradient; stage $6,2.5 \mathrm{mph}$ with $5 \%$ gradient; stage $7,2.5 \mathrm{mph}$ with $7.5 \%$ gradient; stage 8 , 2.5 mph with $10 \%$ gradient; stage 9, $2.5 \mathrm{mph}$ with $12.5 \%$ gradient. Each stage lasted 6 minutes.

Patients were encouraged to exercise until they felt unable to continue. The exercise test was stopped at the patient's request and the reason for stopping was recorded. The ten controls followed both the heart failure protocol and the Bruce protocol to determine their maximum oxygen consumption.
MEASUREMENT OF RESPIRATORY GAS EXCHANGE Oxygen consumption and carbon dioxide production and minute ventilation were determined by the method of Davies et $\mathrm{al}^{16}$ and as described below. Each subject wore a head support and J respiratory valve (Collins) and nose clip. Expired air was sampled from different ports on a seven litre mixing box (Airspec). $100 \%$ argon was added to the inlet port of the mixing box at a known flow rate. We used a mass spectrometer (Centronic Q806) to analyse mixed expired oxygen, nitrogen, carbon dioxide, and argon from the mixing box. The output of the spectrometer was known to be linear ( $<1 \%$ deviation) over the range of gas concentrations measured. The four channel output from the spectrometer was linked to a BBC B computer via the analogue to digital converter within the computer. Minute ventilation (VE) at body temperature, pressure, and saturation (BTPS) was calculated as follows:

$\mathrm{VE}=\mathrm{M}$ argon $\times\left(100-\mathrm{F}_{\text {box }} \mathrm{Ar}\right) \times 0.863 \div\left(\mathrm{F}_{\text {box }}\right.$ $\left.\mathrm{Ar}-\mathrm{F}_{\mathrm{I}} \mathrm{Ar}\right) \times$ (barometric pressure $\left.(\mathrm{mm} \mathrm{Hg})-47\right)$ $1 \mathrm{~min}^{-1}$ BTPS

$\mathrm{M}$ argon $=$ argon flow $\left(\mathrm{ml} \mathrm{min}^{-1}\right) \times$ barometric pressure $(\mathrm{mm} \mathrm{Hg}) \times 273 \div 760 \times(273+$ room temperature $\left({ }^{\circ} \mathrm{C}\right)$ )

where $\mathrm{M}$ argon is argon flow in millilitres (at standard temperature and pressure, dry (STPD)) per minute; $\mathrm{F}_{\text {box }} \mathrm{Ar}$ is percentage concentration of argon in mixing box; and $\mathrm{F}_{\mathrm{I}} \mathrm{Ar}$ is percentage concentration inspired argon $(0.93 \%)$.

Oxygen consumption $\left(\mathrm{V}_{2}\right)$ in millilitres per minute (STPD) was calculated as follows:

$\dot{V O}_{2}=\frac{M \operatorname{argon}\left(100-F_{1} A r\right)}{100\left(F_{\text {box }} A r-F_{1} A r\right)} \times$
$\quad\left[\left(\frac{F_{b o x} N_{2} \times F_{1} O_{2}}{F_{1} N_{2}}\right)-F_{b o x} O_{2}\right]$.

where $\mathrm{F}_{1} \mathrm{O}_{2}$ is percentage inspired oxygen $(20.93 \%)$; $\mathrm{F}_{\mathrm{box}} \mathrm{O}_{2}$ is percentage of oxygen in mixing box; $\mathrm{F}_{\text {box }} \mathrm{N}_{2}$ is percentage of nitrogen in mixing box; $\mathrm{F}_{1} \mathrm{~N}_{2}$ is percentage of inspired nitrogen $(78.09 \%)$.

Carbon dioxide production $\left(\dot{\mathrm{V}} \mathrm{CO}_{2}\right)$ in millilitres per minute (STPD) was calculated as follows:

$$
\dot{\mathrm{VCO}}_{2}=\frac{\mathrm{M} \operatorname{argon}\left(100-\mathrm{F}_{\mathrm{I}} \mathrm{Ar}\right)}{100\left(\mathrm{~F}_{\text {box }} \mathrm{Ar}-\mathrm{F}_{\mathrm{I}} \mathrm{Ar}\right)} \times \mathrm{F}_{\text {box }} \mathrm{CO}_{2}
$$

where $\mathrm{F}_{\mathrm{box}} \mathrm{CO}_{2}=$ percentage carbon dioxide in mixing box.

Respiratory quotient (RQ) was calculated as $\dot{\mathrm{VCO}}_{2} \div \mathrm{VO}_{2}$.

Results for $\mathrm{VO}_{2}$ and anaerobic threshold were expressed per $\mathrm{kg}$ bodyweight unless stated otherwise.

The computer sampled mixed expired gas every 
second and averaged the results for 30 seconds. Graphs of $\dot{\mathrm{VO}}_{2}$ against time, $\dot{\mathrm{V}} \mathrm{O}_{2}$ against $\dot{\mathrm{V}} \mathrm{CO}_{2}$, $\dot{\mathrm{V}} \mathrm{O}_{2}$ against ventilation rate, and $\mathrm{VO}_{2}$ against heart rate were plotted at the end of an experiment.

To validate the measurements of $\mathrm{VE}, \mathrm{VO}_{2}$, and $\dot{\mathrm{V}} \mathrm{CO}_{2}$, expired gas was collected from the exit port of the mixing box into a Douglas bag. The volume of gas collected was measured by a Parkinson-Cowan gas meter and the concentrations of mixed expired gas were analysed with the spectrometer. The calibration of the gas meter was checked with a BOC flow meter. The response time of the equipment was assessed by pumping air into the mixing box with a Rudolph syringe. Sudden changes in "ventilation" ( 0 to 15 litres, 15 to 20 litres, $20-30$ litres, $30-40$ litres, 40-50 litres, 50-60 litres) were made and the time taken to reach $\geqslant 95 \%$ of the final minute ventilation was recorded.

\section{MEASUREMENT OF ANAEROBIC THRESHOLD}

The point at which carbon dioxide production increased disproportionately in relation to oxygen consumption was determined by two methods. It was determined subjectively by three independent observers from a graph plotting $\mathrm{VO}_{2}$ against $\dot{\mathrm{VCO}}_{2}$. A satisfactory statistical technique to determine this point is not available. The following method was chosen. Maximum oxygen consumption was measured. The respiratory quotients for individual points up to $50 \%$ maximum $\mathrm{VO}_{2}(55 \%$ if maximum $\mathrm{V}_{2}<1$ litre) were averaged and two standard deviations were calculated. Subsequent points were looked at in groups of three, that is over a period of 1.5 minutes. If two out of three respiratory quotients fell outside two standard deviations of the mean $R Q$ then the first of the two points was taken and the $\mathrm{VO}_{2}$ at the time was recorded as the anaerobic threshold. Variation in results between observers and methods was analysed.

\section{STATISTICAL ANALYSIS}

Results are quoted as mean and standard error. Interclass differences were assessed by one way analysis of variance and Student's $t$ test. The reproductibility of measures of oxygen consumption and anaerobic threshold was determined by means of the retest reliability coefficient (Rtt):

$\left(\frac{\text { total variance reading } 1 \text { - error variance }}{\text { total variance reading } 1}\right) \times 100 \%$ where error variance equals (reading 1 - reading 2$)^{2}$ $\div$ number of patients.

\section{Results}

Validation of the technique Gas concentrations in three different samples of each gas were measured by spectrometer. The difference between actual concentration and the measured value was $\leqslant 1 \%$.

Douglas bag collections. The argon dilution technique underestimated $\mathrm{VE}$ and subsequent $\mathrm{VCO}_{2}$ and $\mathrm{VO}_{2}$, when compared with Douglas bag measurements for minute ventilation of approximately 10,20 , and 60 litres, by $2 \%, 3 \%$, and $2 \%$ respectively.

Response time to changes in minute ventilation. After sudden changes in ventilation the time taken to reach $95 \%$ of the final value was $\leqslant 1 \mathrm{~min}$.

New York Heart Association (NYHA) and specific activity scale criteria of assessment. Ten patients were NYHA class II, 14 NYHA class III (four NYHA class IIIA, 10 NYHA class IIIB). As judged by the specific activity scale assessment four were class I, six class II, 12 class III, 2 class IV.

\section{Drug therapy}

All patients were taking diuretics-mean dose of frusemide was $60 \mathrm{mg}$ for patients in NYHA class II and $110 \mathrm{mg}$ for those in class III. Vasodilator treatment included captopril (one patient NYHA class II, nine patients NYHA class III), nitrates (one patient NYHA class II, two patients NYHA class III); and nine patients were taking digoxin, seven of them were in sinus rhythm.

\section{Reproducibility of oxygen consumption measurements} All patients underwent at least three exercise tests. After an initial test to familiarise patients with the treadmill maximum oxygen consumption was measured during the second and third exercise tests. The results of these two tests were compared. There was no relation between the error variance and the maximum oxygen consumption on the second exercise test $(r=0 \cdot 08)$. Therefore the group was analysed as a whole.

The retest reliability coefficient for maximum oxygen consumption was $90.5 \%$ for heart failure patients and $89.9 \%$ for controls. The mean maximum oxygen consumption during the third exercise test was $1 \%$ greater than that during the second exercise test (not significant).

In 10 patients (three New York Heart Association class II, three class IIIA, four class IIIB) a fourth exercise test was performed. In eight patients maximum oxygen consumption assessed in the fourth exercise test did not differ by more than $10 \%$ compared with the third exercise test but in two patients it was greater by $16 \%$ and $38 \%$. This difference was associated with increases in exercise duration of $60 \%$ and $42 \%$ respectively. 


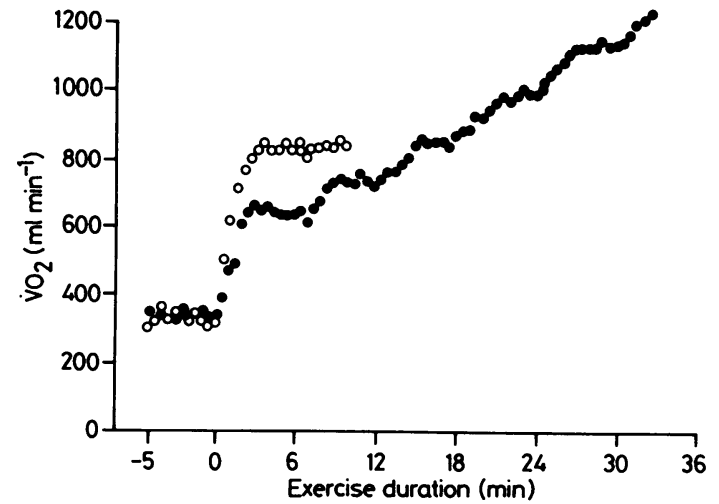

Fig. 1 Oxygen consumption $\left(\mathrm{VO}_{2}\right)$ in relation to duration of exercise in one patient. The graph shows a stepped increase in oxygen consumption during treadmill exercise (O) and a sharp increase in $\mathrm{VO}_{2}$ from resting state to stage 3 of treadmill protocol $(\bigcirc)$. A steady state for oxygen consumption was reached in three minutes.

\section{Reproducibility of anerobic threshold measurements} In five patients the anaerobic threshold could not be assessed because of the scatter of respiratory quotient measurements. There was good correlation among three observers when the anaerobic threshold was assessed by direct inspection $(r=0.99,0.98)$. There was a strong correlation $(r=0.97)$ between the results of direct inspection and those of objective assessment. The retest reliability coefficient for anaerobic threshold measurement for the second and third exercise tests was assessed for direct inspection and objective analyses of the measurement. The Rtt for assessment by direct inspection was $96 \%$ and for objective assessment it was $91 \%$. In the two patients where maximum oxygen consumption differed by $16 \%$ and $38 \%$ for the third and fourth exercise tests the anaerobic threshold measurements differed by only $-9 \%$ and $6 \%$ respectively.

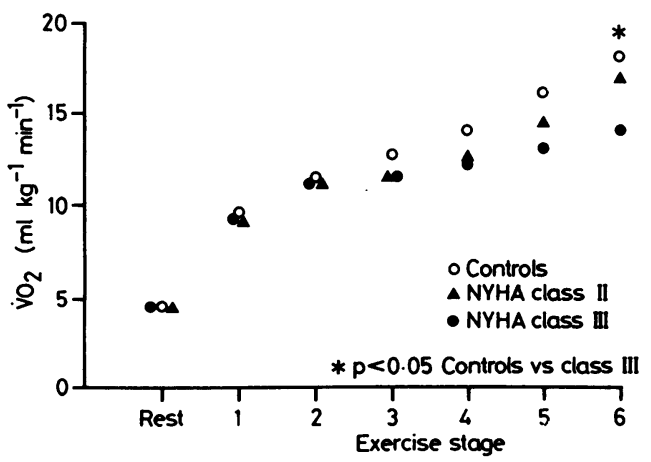

Fig. 2 Relation between oxygen consumption $\left(\mathrm{VO}_{2}\right)$ and exercise stage in controls and patients in New York Heart Association classes II and III.
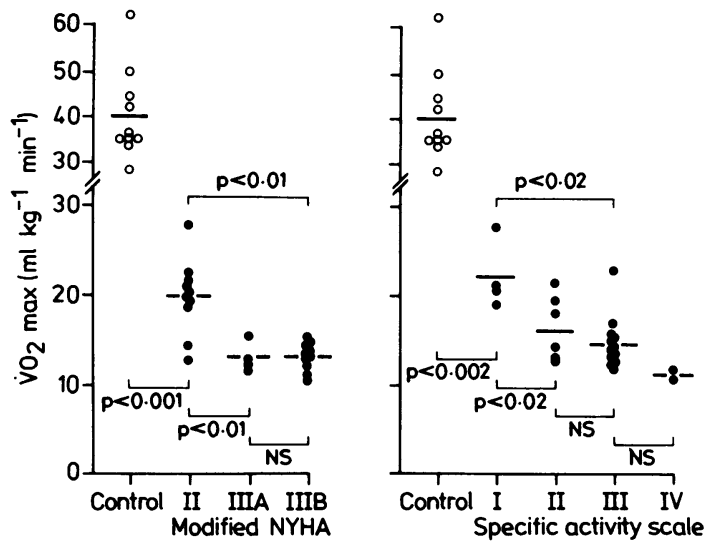

Fig. 3 Oxygen consumption in controls and cardiac patients. Relation between maximum oxygen consumption $\left(\mathrm{VO}_{2}\right.$ max) and functional class according to modified New York Heart Association (NYHA) class specific activity scale. Means are indicated.

Oxygen consumption with increasing workload

No patient reached a plateau of oxygen consumption with increasing workload (oxygen consumption increase of $\leqslant 1 \mathrm{ml} \mathrm{kg}^{-1} \mathrm{~min}^{-1}$ over previous workload $)^{3}$ despite repeat exercise testing. After each change in workload, oxygen consumption reached a peak for that workload within three minutes (Fig. 1). At submaximal workloads oxygen consumption was similar in controls and in patients with heart failure, but it was lower in New York Heart Association class III patients who completed stage 6 compared with controls at the same treadmill state $(p<0.05)$ (Fig. 2).

Maximum oxygen consumption related to functional class

There was a significant difference in maximum oxygen consumption $\left(\mathrm{ml} \mathrm{kg}^{-1} \mathrm{~min}^{-1}\right)$ between functional classes (Fig. 3). Maximum oxygen consumption results for the controls and patients in each New York Heart Association class were as follows: controls $40 \cdot 1(3 \cdot 1)$, class II $19 \cdot 7(1 \cdot 4)$, class IIIA $13 \cdot 3(1 \cdot 1)$, class IIIB $13 \cdot 1(0 \cdot 52)$. The following differences were tested for statistical significance: controls vs New York Heart Association class II ( $p<0.0001)$; II vs IIIA ( $p<0.01)$; and IIIA vs IIIB (not significant). Maximum oxygen consumption for each specific activity scale class were as follows: I, $22.0(1.9)$; II, 16.0(1.6); III, 14.4(0.83); IV $10 \cdot 7(0.54)$. The following differences were tested for statistical significance: controls $v s$ specific activity scale class I $(\mathrm{p}<0.002)$; I vs II $(\mathrm{p}<0.02)$; II vs III (not significant); and III vs IV (not significant). 


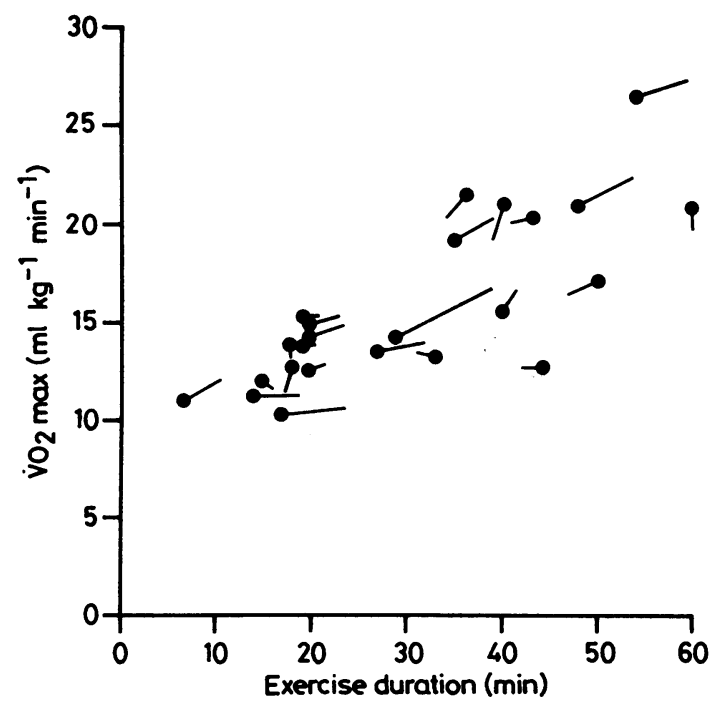

Fig. 4 Exercise duration in cardiac patients. Relation between maximum oxygen consumption ( $\mathrm{VO}_{2}$ max) and exercise duration. The change in exercise duration and maximum oxygen consumption between exercise tests 2 and 3 is shown. The dot represents result of exercise test 2 and the line represents the change between test 2 and test 3.

Maximum oxygen consumption related to exercise duration

Maximum oxygen consumption correlated with exercise duration though there was considerable variation in exercise duration between patients with similar maximum oxygen consumptions (Fig.4).

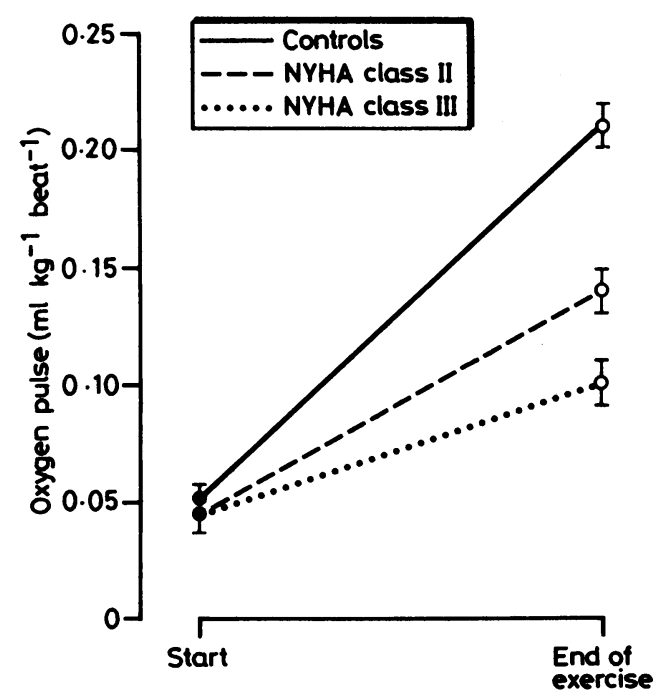

Fig. 5 Oxygen pulse at start and end of exercise and its relation to New York Heart Association (NYHA) functional class.
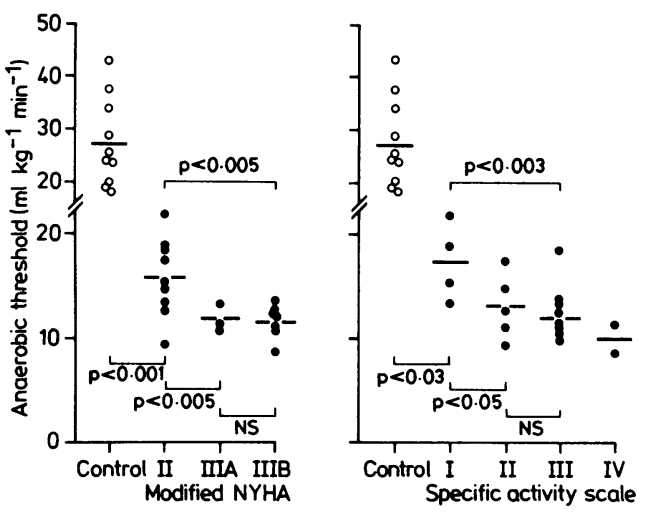

Fig. 6 Anaerobic threshold in normal subjects and cardiac patients and its relation to functional class according to modified New York Heart Association criteria and specific activity scale. Mean values are indicated.

Although there was no significant difference in maximum oxygen consumption between exercise tests 2 and 3, exercise duration was longer in exercise test 3 (Fig. 4) (31 vs 26 minutes, $\mathrm{p}<0.05$ ).

Maximum oxygen consumption related to heart rate There were significant differences in oxygen pulse (oxygen consumption divided by heart rate) ${ }^{17}$ at the end of exercise between controls and New York Heart Association class II patients $(p<0.002)$ and class II and class III patients ( $p<0.03)$ (Fig. 5).

Anaerobic threshold related to functional class

Anaerobic thresholds $\left(\mathrm{ml} \mathrm{kg}^{-1} \mathrm{~min}^{-1}\right)$ for controls and for patients in each New York Heart Association class were as follows (Fig. 6): controls, 27.5(3.0); II, $15 \cdot 7(1 \cdot 2)$; IIIA, $11 \cdot 7(0 \cdot 7)$; IIIB, 11.3(0.4). The following differences were tested for statistical significance: controls vs New York Heart Association class II ( $\mathrm{p}<0.001)$; II vs IIIA ( $\mathrm{p}<0.007)$; IIIA vs IIIB (not significant). Anaerobic thresholds for patients in each specific activity scale class were as follows: I, 17.3(1.0); II, 13.0(0.7); III, 11.8(0.5), IV, $9 \cdot 5(0.5)$. The following differences were tested for statistical significance: controls vs specific activity scale class I ( $<<0.03)$; I vs II ( $<<0.05)$; II vs III (not significant).

Exercise duration related to functional class

Patients exercised for a shorter time as the degree of their functional impairment increased, though there was considerable variation (Fig. 7). The exercise duration of patients in various New York Heart Association classes was as follows: II, 39.7(3.3); IIIA, 27.6(5); IIIB, 19.1(2.9). The exercise duration of patients in specific activity scale classes were as follows: I, 44.3(3.5); II, 30.2(4.8); III, 24.9(3.2); IV, 

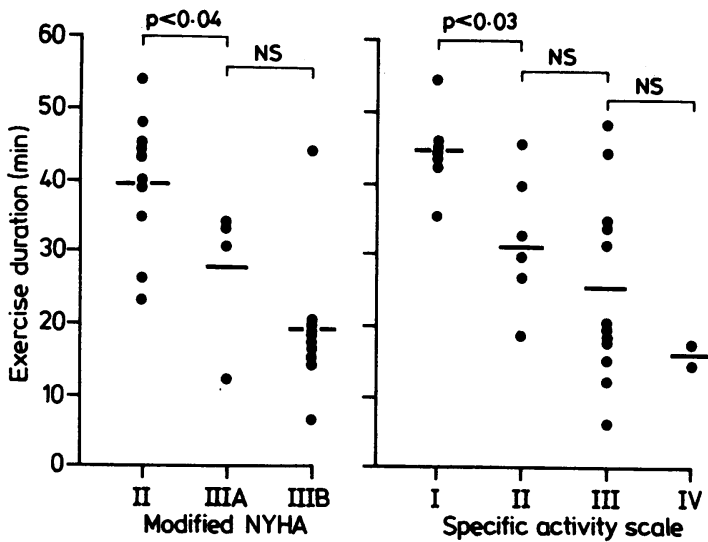

Fig. 7 Exercise duration in cardiac patients and its relation to functional class according to modified New York Heart Association criteria and specific activity scale. Mean values are indicated.

15.5(1.3).The differences between New York Heart Association classes II and IIIA $(p<0.04)$ and specific activity scale classes I and II $(p<0.03)$ were statistically significant.

\section{Discussion}

The severity of heart failure is usually graded according to the level of physical activity at which dyspnoea and fatigue appear. A more objective analysis is desirable in order to classify patients with heart failure and to assess the efficacy of treatment. Measurement of respiratory gas exchange may provide a non-invasive means of assessing functional capacity and cardiac reserve. ${ }^{3467}$

In highly motivated normal subjects oxygen consumption increases with increasing workload and then reaches a definite plateau. ${ }^{18}$ The plateau represents the physiological limit of oxygen consumption and is a measure of the total aerobic capacity of the individual. Weber et al noted a similar response to treadmill exercise in patients with heart failure. ${ }^{3}$ In the present study no such plateau was demonstrated despite patients being encouraged to continue the test until their symptoms became uncomfortable (Fig. 1). Maximum oxygen consumption was therefore symptom limited in these patients. There are two explanations for this difference between studies. First, the protocol used by Weber $e^{-a^{3}}$ may have produced an artificial plateau effect because the increments in workload at the end of the protocol were smaller than those at the beginning. Second, oxygen distribution in the body may not have equilibrated in the final stage of exercise. In the present study it took three minutes before oxygen con- sumption reached a steady state after a change in workload (Fig. 1). Consequently if the exercise test continued for less than three minutes at the final workload oxygen consumption would appear to have reached a plateau. Oxygen consumption probably failed to reach a plateau in our patients because they stopped exercising when symptoms began rather than when oxygen consumption reached its limit.

Maximum oxygen consumption was related to functional class as assessed by the New York Heart Association and specific activity scale criteria and there were statistically significant differences between some classes (Fig. 3). In other studies there was no significant difference in oxygen consumption between New York Heart Association classes II and III. ${ }^{38}$ The definition of New York Heart Association class II that we used was not the same as the original definition $^{14}$ that was used by Weber et $a l^{3}$ and Franciosa ${ }^{8}$ and this probably accounts for the differences between studies.

In most individuals there was little change in maximum oxygen consumption when exercise testing was repeated over several days. Although maximum oxygen consumption in tests 2 and 3 was not significantly different, exercise duration and hence measured workload was significantly greater in test 3 because patients had became accustomed to walking on the treadmill. This result demonstrates that a change in treadmill performance is not a reliable indicator of a change in cardiorespiratory reserve.

Oxygen consumption generally was similar in controls and in patients with heart failure, but it was lower in New York Heart Association class III patients who completed stage 6 than in controls at the same treadmill stage (Fig. 2). This finding is consistent with other studies. ${ }^{219}$ Cowley et al, however, reported large differences in oxygen consumption between controls $(50 \%$ higher) and patients with heart failure who exercised at a workload comparable to stage I of our protocol. ${ }^{20}$ The difference occurred at the first stage of exercise and remained almost constant despite increasing exercise loads. A possible explanation is that their controls had not practiced on the treadmill and that anxiety and poor treadmill walking technique affected measured oxygen consumption. These factors may also explain the scatter of results for exercise duration and oxygen consumption noted in our study (Fig. 4).

If measurement of maximum oxygen consumption is to be useful in the assessment of patients with heart failure it must reflect cardiac status. Bias due to motivation of the patient is inherent in the measurement of maximum oxygen consumption. Measurement of anaerobic threshold may be a more objective method of assessing functional capacity. 
Respiratory gas exchange analysis has been used to identify the aerobic/anaerobic threshold in patients with heart failure. ${ }^{2122}$ The threshold is reached when there is a disproportionate rise in carbon dioxide production relative to oxygen consumption. This point occurs at approximately $60 \%$ of maximum oxygen consumption ${ }^{10}$ and before exercise is ended by the patient. The physiological importance of the anaerobic threshold is uncertain but the threshold is associated with a rise in plasma lactate ${ }^{23}$ and will occur when the rate of lactate production exceeds the rate of its metabolism. Plasma lactate increases during anaerobic metabolism as a consequence of an inadequate oxygen supply to active tissue $\mathrm{e}^{24}$ and lactate is metabolised mainly in the liver. In heart failure lactate metabolism may be reduced because of impaired hepatic blood flow and hepatic congestion. In rats training increased the anaerobic threshold not by reducing lactate production but by increasing lactate clearance. ${ }^{25}$ Thus, the lower anaerobic threshold observed in patients with severe heart failure may reflect both diminished blood flow to exercising muscle and reduced lactate clearance.

In the present study the anaerobic threshold could easily be determined by respiratory gas measurement in most patients, but in $21 \%$ of patients the scatter of respiratory quotient measurements prevented assessment by this method. Irregular breathing patterns resulted in transient fluctuations in expired carbon dioxide levels. This effect was seen in most patients at the start of exercise but persisted in some patients throughout the test. To minimise this artefact respiratory gas measurements made during the first minute of exercise were excluded from the assessment of the anaerobic threshold. In individual patients, repeated exercise testing reduced the scatter in the respiratory quotients measurements suggesting that anxiety was partly responsible. The effect was most pronounced in patients with the poorest exercise tolerance.

As expected the anaerobic threshold was lowest in the patients with more severe heart failure, though there was considerable overlap between functional classes (Fig. 6) as was also reported by Matsumura et al. ${ }^{22}$

Any change in functional class in an individual patient would be expected to be accompanied by a change in anaerobic threshold. When maximum oxygen consumption alters without a change in the anaerobic threshold a change in cardiac status is questionable. In two patients maximum oxygen consumption increased by $16 \%$ and $39 \%$ in successive exercise tests yet the anaerobic threshold changed by $-6 \%$ and $+9 \%$ respectively and this suggests that a change in functional class had not occurred, but rather that patient motivation had altered between the two tests.

In conclusion, maximum oxygen consumption and anaerobic threshold can be reliably measured in patients with impaired ventricular function. In most patients measurement of maximum oxygen consumption is reproducible but since it is partly determined by patient motivation it may change in the absence of a change in cardiac function. Measurement of maximum oxygen consumption alone can be misleading, but together with knowledge of the anaerobic threshold and exercise duration it provides an objective and accurate means of classifying and following patients with heart failure.

\section{References}

1 Patterson JA, Naughton J, Pietras RJ, Gunnar RM. Treadmill exercise in assessment of the functional capacity of patients with cardiac disease. Am $\mathcal{F}$ Cardiol 1972; 30: 757-62.

2 Franciosa JA, Ziesche S, Wilen M. Functional capacity of patients with chronic left ventricular failure. $A m \mathcal{F}$ Med 1979; 67: 460-6.

3 Weber KT, Kinasewitz GT, Janicki JS, Fishman AP. Oxygen utilization and ventilation during exercise in patients with chronic cardiac failure. Circulation 1982; 65: 1213-23.

4 Franciosa JA, Park M, Levin TB. Lack of correlation between exercise capacity and indexes of resting left ventricular performance in heart failure. $\mathrm{Am} \mathcal{F}$ Cardiol 1981; 47: 33-9.

5 Roskamm H, Schnellbacher L, Samek L, Betz P. Does exercise testing with invasive measurements of cardiac output and pressure really contribute? Eur Heart $\mathcal{F}$ 1983; 4 (suppl A): 127-30.

6 Franciosa JA, Leddy CL, Wilen M, Schwartz DE. Relation between hemodynamic and ventilatory responses in determining exercise capacity in severe congestive heart failure. Am $\mathcal{F}$ Cardiol 1984; 53: 127-34.

7 Wilson JR, Martin JL, Ferraro N. Impaired skeletal muscle nutritive flow during exercise in patients with congestive heart failure: role of cardiac pump dysfunction as determined by the effect of dobutamine. $\mathrm{Am}$ f Cardiol 1984; 53: 1308-15.

8 Franciosa JA. Exercise testing in chronic congestive heart failure. $A m \mathcal{F}$ Cardiol 1984; 53: 1447-50.

9 Hollmann WA. Zur Frage der Daverleistungstähigkeir. Fortschr Med 1961; 77: 439-53.

10 Wasserman K, Whipp BJ, Koyal SN, Beaver WL. Anaerobic threshold and respiratory gas exchange during exercise. 3 Appl Physiol 1973; 35: 236-43.

11 Wasserman K, McIlroy MB. Detecting the threshold of anaerobic metabolism in cardiac patients during exercise. $\mathrm{Am}$ f Cardiol 1964; 14: 844-52.

12 Wasserman K, Whipp BJ. Exercise physiology in health and disease. Am Rev Respir Dis 1975; 112: 219-49.

13 Koyal SN, Whipp BJ, Huntsman D, Bray GA, Wasserman $\mathrm{K}$. Ventilatory responses to the metabolic acid- 
osis of treadmill and cycle ergometry. $\mathcal{F}$ Appl Physiol 1976; 40: 864-7.

14 The Criteria Committee of the New York Heart Association. Diseases of the heart and blood vessels. Nomenclature and criteria for diagnosis. 6 th ed. Boston: LittleBrown, 1964.

15 Goldman L, Hashimoto B, Cook EF, Loscalzo A. Comparative reproducibility and validity of systems for assessing cardiovascular functional class: advantages of a new specific activity scale. Circulation 1981; 64: 1227-34.

16 Davies NJ, Dennison DM. The measurement of metabolic gas exchange and minute volume by mass spectrometry alone. Respir Physiol 1979; 36: 261-7.

17 Conn EH, Williams RS, Wallace AC. Exercise responses before and after physical conditioning in patients with severely depressed left ventricular function. Am f Cardiol 1982; 49: 296-300.

18 Astrand PO. Measurement of maximum aerobic capacity. Can Med Assoc f 1967; 96: 732-5.

19 Wilson JR, Martin JL, Schwartz D, Ferraro N. Exercise intolerance in patients with chronic heart failure: role of impaired nutritive flow to skeletal muscle. Circu- lation 1984; 69: 1079-87.

20 Cowley AJ, Rowley JM, Stainer K, Hampton JR. Effects of captopril on abnormalities of the peripheral circulation and respiratory function in patients with severe heart failure. Lancet 1984; ii: 1120-4.

21 Wilson JR, Ferraro N, Weber KT. Respiratory gas analysis during exercise as a noninvasive measure of lactate concentration in chronic congestive heart failure. Am f Cardiol 1983; 51: 1639-43.

22 Matsumura N, Nishijima H, Kojima S, Hashimoto F, Minami M, Yasuda $H$. Determination of anaerobic threshold of assessment of functional state in patients with chronic heart failure. Circulation 1983; 68: 360-7.

23 Hermansen L. Lactate production during exercise. In: Pernow B, Saltin B, eds. Muscle metabolism during exercise. New York: Plenum, 1971: 401-7.

24 Karlsson J, Jacobs I. Onset of blood lactage accumulation during muscular exercise as a threshold concept. Theoretical considerations. Int I Sports Med 1982; 3: 190-201.

25 Donovan CM, Brooks GA. Endurance training affects lactate clearance, not lactate production. Am $\mathcal{f}$ Physiol 1983; 244: E83-92. 